\title{
Pregabalin and placebo responders show different effects on central pain processing in chronic pancreatitis patients
}

This article was published in the following Dove Press journal: Journal of Pain Research

10 July 2015

Number of times this article has been viewed

\section{Stefan AW Bouwense' \\ Søren S Olesen² \\ Asbjørn M Drewes ${ }^{2}$ \\ Harry van Goor' \\ Oliver HG Wilder-Smith ${ }^{3}$}

'Pain and Nociception Neuroscience Research Group, Department of Surgery, Radboud university medical center, Nijmegen, The Netherlands; ${ }^{2}$ Mech-Sense, Department of Gastroenterology and Hepatology, Aalborg University Hospital, Aalborg, Denmark; ${ }^{3}$ Pain and Nociception Neuroscience Research Group, Department of Anaesthesiology, Pain and Palliative Medicine, Radboud university medical center, Nijmegen, The Netherlands

Correspondence: Oliver HG WilderSmith

Pain and Nociception Neuroscience Research Group, Department of Anaesthesiology, Pain and Palliative Medicine, Radboud university medical center, PO Box 9101/520,

6500 HB Nijmegen, The Netherlands

$\mathrm{Tel}+3 \mathrm{I} 243668120$

Fax +3। $2436 \mid 3585$

Email oliver.wilder-smith@radboudumc.nl
Background: Pain control in chronic pancreatitis is a major challenge; the mechanisms behind analgesic treatment are poorly understood. This study aims to investigate the differences in pain sensitivity and modulation in chronic pancreatitis patients, based on their clinical response (responders vs nonresponders) to placebo or pregabalin treatment.

Methods: This study was part of a randomized, double-blind, placebo-controlled trial evaluating the analgesic effects of pregabalin and placebo in chronic pancreatitis. Post hoc, patients were assigned to one of four groups, ie, responders and nonresponders to pregabalin $(n=16 ; n=15)$ or placebo $(n=12 ; n=17)$ treatment. Responders were defined as patients with $>30 \%$ pain reduction after 3 weeks of treatment. We measured change in pain sensitivity before and after the treatment using electric pain detection thresholds (ePDT) in dermatomes C5 (generalized effects) and Ventral T10 (segmental effects). Descending endogenous pain modulation was quantified via conditioned pain modulation (CPM) paradigm.

Results: Sixty patients were analyzed in a per-protocol analysis. ePDT change in C5 was significant vs baseline and greater in pregabalin $(1.3 \mathrm{~mA})$ vs placebo responders $(-0.1 \mathrm{~mA}$; $P=0.015)$. This was not so for ePDT in Ventral T10. CPM increased more in pregabalin (9\%) vs placebo responders $(-17 \% ; P<0.001)$. CPM changed significantly vs baseline only for pregabalin responders $(P=0.006)$.

Conclusion: This hypothesis-generating study provides the first evidence that pain relief with pregabalin is associated with anti-hyperalgesic effects and increased endogenous inhibitory modulation. No such effects were observed in patients experiencing pain relief with the placebo treatment. The mechanisms underlying analgesic response to placebo vs drug treatments are different and, together with their interactions, deserve further study.

Keywords: chronic pancreatitis, pregabalin, placebo, chronic pain treatment, quantitative sensory testing, central sensitization

\section{Introduction}

Morphological changes of the pancreas due to chronic pancreatitis may cause intense pain, necessitating surgical or endoscopic intervention. However, many patients remain symptomatic even after successful surgical interventions. ${ }^{1,2}$ The majority of these patients have a long history of treatments with opioid-based analgesic medications with limited effectiveness and undesirable side effects. ${ }^{3}$ A good explanation for treatment failure in these patients is lacking to date. Hence, to gain a better control of pain, more evidence is needed on the origin of chronic pain and how this is influenced by pain treatment.

We performed a randomized clinical trial to evaluate the effect of pregabalin or placebo on pain in patients with chronic pancreatitis. We showed better pain relief in 
the pregabalin group than in the placebo group. Interestingly, a significant pain reduction on average pain could also be observed in the placebo group. ${ }^{4}$ Secondary analysis of this trial confirmed that patients with chronic pancreatitis showed signs of altered central pain processing, which was related to disease stage, ${ }^{5}$ and inhibitory effects on central sensitization by pregabalin. ${ }^{6}$ The impact and the magnitude of placebo analgesia has been described in recent studies and meta-analyses, and is evolving from experimental pain to clinical pain. ${ }^{7-10}$ Analysis of the placebo effect has shown that antinociceptive placebo effects may be mediated by opioid receptor ligands and that specific brain areas are involved. ${ }^{11-13}$ However, more information is needed regarding the mechanisms involved in possible anti-hyperalgesic and analgesic placebo effects.

Our trial also showed that some patients experienced large pain reduction with pregabalin or placebo (responders), whereas some experienced hardly any pain reduction (nonresponders). ${ }^{14}$ In animal models, the variability in response to pain treatment was related to the activity of different pain pathways, eg, the inhibitory $\gamma$-aminobutyric acid-ergic system, or activation of excitatory N-methyl-D-aspartate receptors in the central nervous system. ${ }^{15-17}$ Another explanation might be that ongoing nociceptive input due to the inflammation of the pancreas produces changes in central pain processing, which are no longer dependent on ongoing peripheral nociceptive input. ${ }^{18,19}$ Exploration of basic pain mechanisms in responder and nonresponder groups by quantitative sensory testing (QST) might also help us understand the variable clinical response to pain and placebo treatment between individual patients.

The aim of this hypothesis-generating study was to investigate the difference in pain sensitivity and modulation by QST in patients with chronic pancreatitis who were responders and nonresponders to placebo or pregabalin treatment. This study is a secondary analysis of our original randomized controlled trial; ${ }^{4}$ the population and the design are similar to one of our previous studies. ${ }^{14}$

\section{Methods}

\section{Study overview}

This study was part of an investigator-initiated, doubleblind, placebo-controlled, parallel-group study of increasing doses of pregabalin or placebo conducted in the Netherlands (Department of Surgery, Radboud university medical center) and Denmark (Department of Gastroenterology and Hepatology, Aalborg University Hospital). The study was approved by the Ethical Committees in both the countries (CMO region Arnhem-Nijmegen, Nijmegen, the Netherlands and The local Ethics Committee North Region, Aalborg,
Denmark), and all patients provided written informed consent. This article presents a secondary and further analysis of the data obtained in a previous trial focusing primarily on experimental (QST) end points. ${ }^{4}$ A further explanation on how this study relates to our trial and other sub-studies we performed can be found as Supplementary material.

\section{Patients}

For trial inclusion, patients needed to have chronic abdominal pain typical for pancreatitis (ie, dull epigastric pain more than 3 days per week for at least 3 months) and a diagnosis of chronic pancreatitis based on the Mayo Clinic diagnostic criteria. ${ }^{20}$ The use of a stable regime of concomitant analgesic medication during the trial was another inclusion criterion. Exclusion criteria were painful conditions other than chronic pancreatitis, active (or history of) major depression, allergy to pregabalin or any of its components, an abnormal electrocardiogram at screening visit, severe renal impairment, and pregnant or lactating patients. Only the patients completely complying with the described treatment protocol were analyzed in the present per-protocol analysis.

More details regarding clinical end points, ie, pain scores and side effects, are provided in the original manuscript of the main study. ${ }^{4}$

\section{Randomization and treatment}

The randomization and study procedures have been described in detail in the original study. ${ }^{4}$

The study consisted of a 3-week study period of pregabalin or placebo treatment. During the study period, patients received either escalating doses of pregabalin (300-600 mg/day) or placebo. In the case of unacceptable side effects, a single downward dose titration was allowed. When patients reached their final dosage, they had to stay on that regime for the remaining study period. Patients and those administrating study medication, assessing outcomes, and analyzing data were blinded to group assignment.

For the whole study period, patients were instructed not to change their daily pain medication. Extra pain medication was only allowed in the case of a painful exacerbation of their chronic pancreatitis.

Patients wrote down their average and maximum pain on a visual analog scale (VAS) in a pain diary, where $0=$ no pain and $10=$ worst pain imaginable.

\section{Study visits}

Eligible patients were randomized for placebo or pregabalin on their second visit, 1 week after their screening visit. After randomization, all patients had a baseline QST measurement, 
followed by another QST measurement at the end of the study period of 3 weeks, ie, before they were instructed to taper their medication.

\section{Quantitative sensory testing}

QST took place using a standard temporal test sequence. Testing in females was not standardized with regard to phase of the menstrual cycle because all female patients were amenorrheic. After initial training, electric pain detection thresholds (ePDT) to electric constant current skin stimulation (Digistim; Biometer A/S, Copenhagen, Denmark; tetanic stimulation at $100 \mathrm{~Hz}, 0.2 \mathrm{~ms}$ square waves, self-adhesive electrodes $3 \mathrm{~cm}$ apart) were measured on the dominant side of the body at the following sites: lower neck (C5 dermatome) and pancreatic site (Ventral T10 dermatome) (Figure 1). The Ventral T10 dermatome was chosen because painful stimuli delivered on this skin area are likely to be processed by the same dorsal horn neurons onto which the nociceptive stimuli coming from the pancreas converge. The $\mathrm{C} 5$ dermatome was chosen as a dermatome distant from the pancreas to observe generalized effects on pain thresholds. ${ }^{18,21}$

The conditioned pain modulation (CPM, previously known as diffuse noxious inhibitory controls) paradigm was performed to test the ability of a patient to generate descending inhibitory pain modulation. ${ }^{22,23}$ Pressure pain tolerance thresholds (the test stimulus) were determined before and after the cold pressor task (the conditioning stimulus), and the CPM effect was determined in the nondominant $\mathrm{L} 4 \mathrm{der}$ matome (quadriceps muscle $5 \mathrm{~cm}$ proximal to the patella) as the relative change (\%) in pressure pain tolerance thresholds. For the cold pressor task, the dominant hand was immersed in ice-chilled water $\left(1.0^{\circ} \mathrm{C} \pm 0.3^{\circ} \mathrm{C}\right)$ continuously stirred by a pump. The patient was told to remove the hand from the water after 2 minutes of immersion, or sooner if the pain was considered to be intolerable, and the immersion time noted. Immediately after the cold pressor task, the subjects rated the pain experienced during the test by using a VAS for quality control purposes. ${ }^{18}$

\section{Outcome measures}

Changes in the following parameters (baseline values vs values after 3 weeks of the study medication) were the end points of our study:

1. ePDT for dermatomes $\mathrm{C} 5$ and Ventral T10

2. CPM response.

We chose ePDT as a QST end point for altered pain sensitivity based on publications describing QST measurements before and after pregabalin treatment. These publications showed a strong ePDT response when measurements were

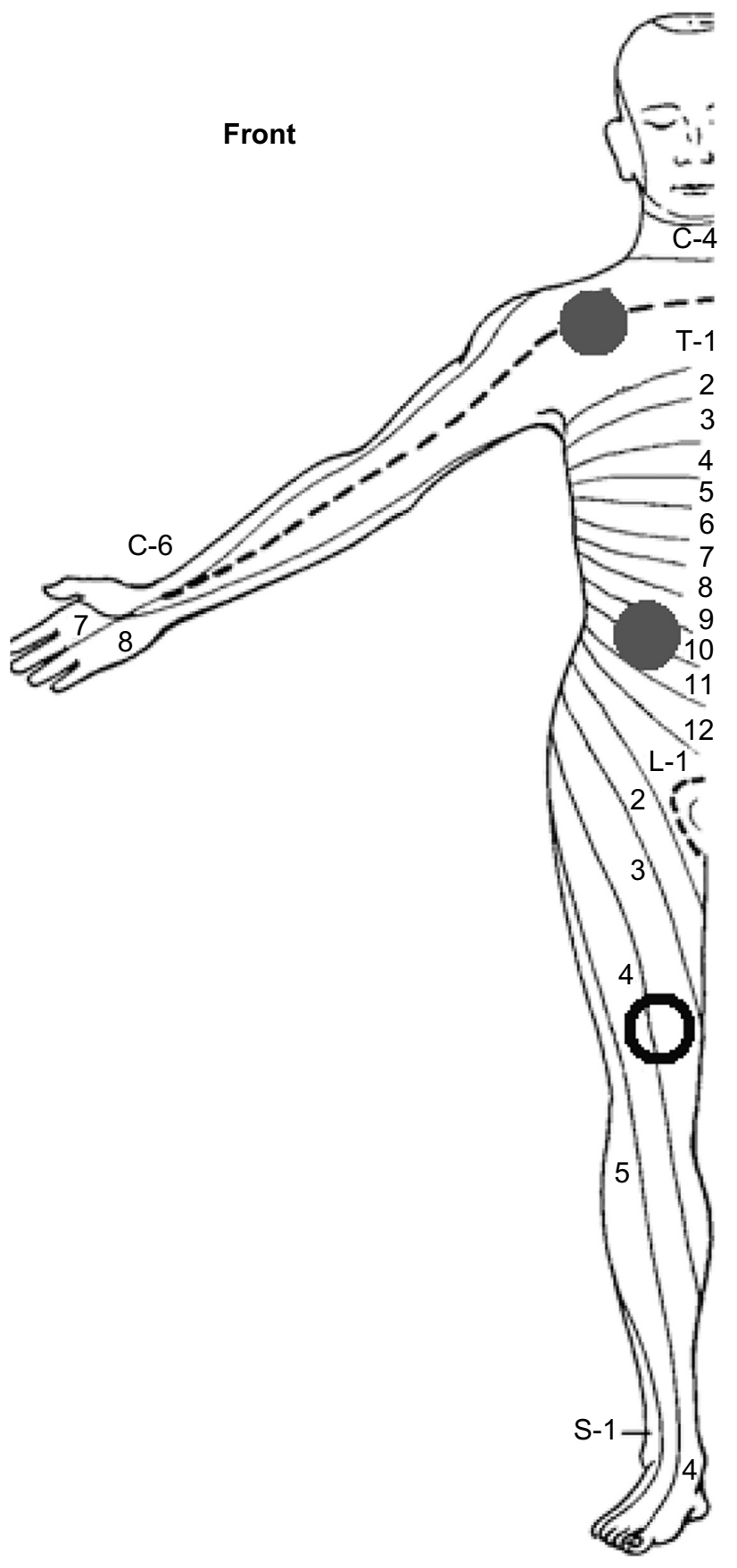

Figure I Dermatomes of measurement for quantitative sensory testing.

Notes: Quantitative sensory testing was performed on the following sites on the dominant body side (closed dots): lower neck (C5 dermatome) and upper abdominal area (Ventral TI0 dermatome). Conditioned pain modulation was determined in the nondominant L4 dermatome (open dot).

compared before and after pregabalin treatment in chronic pancreatitis. ${ }^{6,14}$

\section{Patient groups}

Patients in the pregabalin and placebo groups with more than $30 \%$ pain reduction on their average daily VAS score after 3 weeks of study medication vs baseline values were defined 
as responders. Patients with $30 \%$ or less pain reduction on their average daily VAS score after 3 weeks of study medication were defined as nonresponders. The cut-off point was based on clinical pain studies using a numeric rating scale or VAS. 4,14,24,25 On the basis of study medication and pain reduction, four treatment groups of patients were thus defined: 1) responder placebo group, 2) nonresponder placebo group, 3) responder pregabalin group, and 4) nonresponder pregabalin group.

\section{Statistical analysis}

A pre hoc power calculation based on QST as an end point was not performed because the study was a part of a randomized clinical trial that investigated pregabalin, powered for a clinical primary end point, ie, change in clinical pain score. For this mechanistic study, we performed a per-protocol analysis.

All baseline characteristics and measurements are given as medians with interquartile ranges (IQRs), unless mentioned otherwise.

We performed statistical analysis using Statistica for Windows Software Package (Release 7.0; StatSoft Inc., Tulsa, OK, USA). Statistical significance was set at $P \leq 0.05$.

Baseline characteristics were compared between the groups with Kruskal-Wallis one-way analysis of variance with post hoc analysis using Mann-Whitney $U$ test.

Analysis of incidence of responders and nonresponders within the population was performed by chi-square test.

Within the four treatment groups, baseline values and values after 3 weeks of study medication were analyzed by Wilcoxon signed-rank test for ePDT in the individual dermatomes and CPM response.

Among the four treatment groups, baseline values and their change after 3 weeks' treatment were compared using the Kruskal-Wallis one-way analysis of variance with post hoc analysis using Mann-Whitney $U$ test for ePDT in the individual dermatomes and CPM response. Four subgroups comparisons were performed: 1) placebo responders vs placebo nonresponders, 2) placebo responders vs pregabalin responders, 3) placebo nonresponders vs pregabalin nonresponders and 4) pregabalin responders vs pregabalin nonresponders.

Subgroup (post hoc) analysis was conservatively Bonferroni corrected for multiple comparisons (three comparisons, $P \leq 0.016)$.

\section{Results}

\section{Enrollment and baseline characteristics}

From October 2008 to May 2010, a total of 236 patients were screened and 64 patients were randomized. Sixty patients completed the study according to the treatment protocol and were included in the final analysis (Figure 2). All the patients ( 23 women, 37 men; median age 53 years [IQR 46-62]) had pain due to chronic pancreatitis and were on a stable analgesic therapy. The average daily VAS score of all the patients before the start of trial medication was 4 (IQR 2-5), and their maximum daily VAS score was 5 (IQR 4-7). Their median opioid consumption was $60 \mathrm{mg}$ (IQR 9-146) of morphine equivalents per day. Demographic data of all the four patient groups are provided in Table 1. The data showed no significant differences, except for VAS after 3 weeks of study treatment.

\section{Pain characteristics}

The medians of the average and maximum VAS scores in the pain diaries were comparable at baseline for all (four) the treatment groups. Moreover, the pain medication and morphine equivalents per day were comparable at baseline. The incidence of responders and nonresponders did not differ significantly among all 60 patients and within the placebo or pregabalin group (Table 1).

Within the treatment groups, both the responder groups showed a decline in the average and maximum VAS score after 3 weeks of study treatment (less pain); this was significant for the responder placebo group for average $(P=0.003)$ and maximum $(P=0.003)$ VAS scores and for the pregabalin responder group for average $(P=0.001)$ and maximum $(P=0.001)$ VAS scores (Table 1$)$. The median percentage reduction in average VAS score after 3 weeks of study treatment was $75 \%$ (IQR 54-100) for the placebo responder group and 69\% (IQR 48-94) for the pregabalin responder group. Among all the treatment groups, these results were significantly different compared with the nonresponder placebo and pregabalin groups $(P \leq 0.001)$ (Table 1).

\section{ePDT in individual dermatomes}

At baseline, ePDT in the C5 and Ventral T10 dermatomes was comparable between the treatment groups. Within the treatment groups, only pregabalin responders showed a significant increase in ePDT (less hyperalgesia following pregabalin treatment) for the $\mathrm{C} 5$ dermatome $(P=0.009)$ and Ventral T10 dermatome ( $P=0.009)$ (Table 2$)$.

C5 dermatome (widespread hyperalgesia): Changes in ePDT for the C5 dermatome were significantly different among the four treatment groups overall $(H=10.63, P=0.014)$. Post hoc analysis showed that the pregabalin responder group differed significantly (less hyperalgesia following pregabalin 


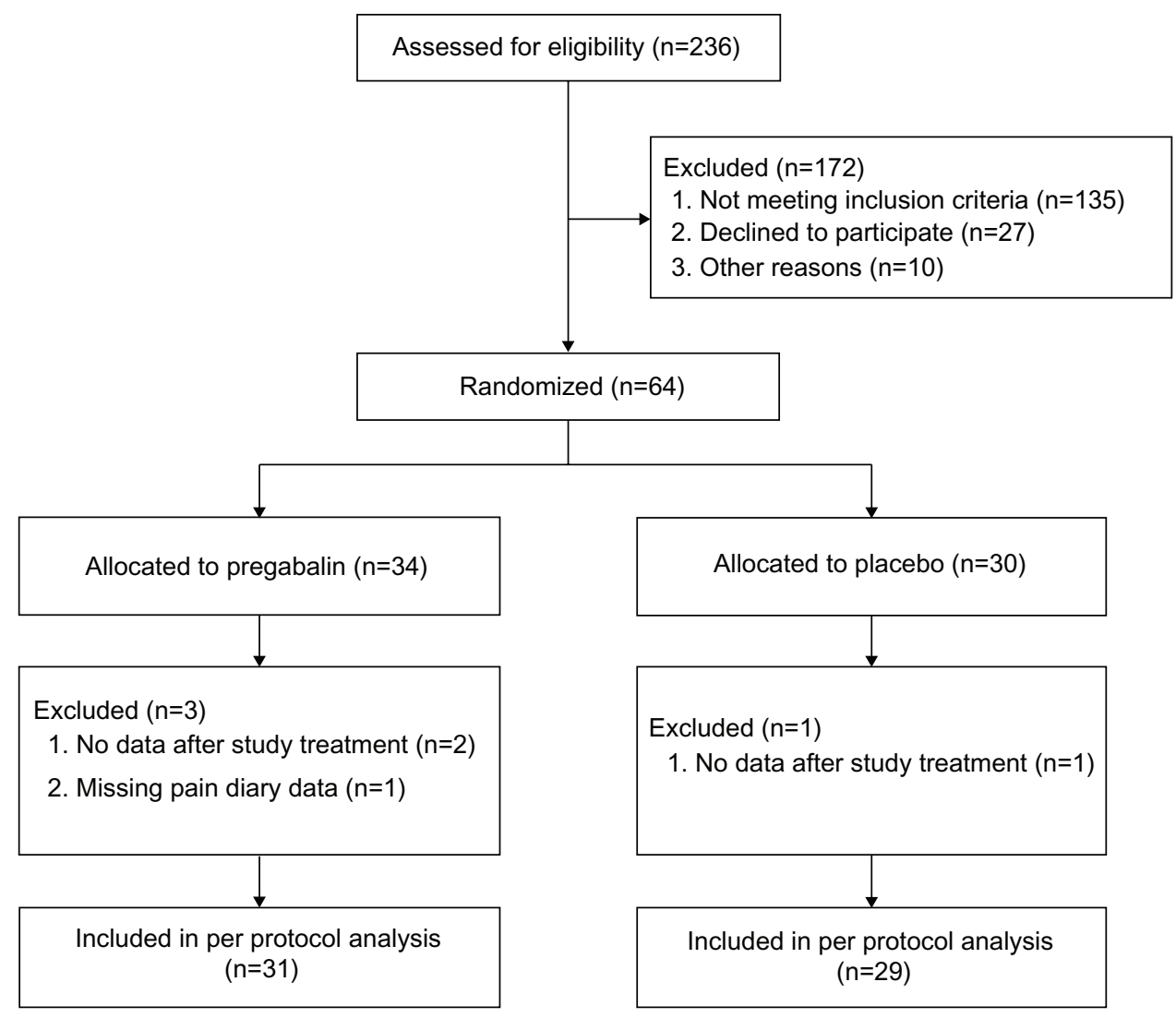

Figure 2 Study enrollment and randomization.

Note: The majority of patients "not meeting inclusion criteria" had died, were pain free, or were no longer being treated in either of the hospitals.

treatment) from the placebo responder group $(P=0.015)$ (Table 2 and Figure 3).

Ventral T10 dermatome (segmental hyperalgesia): No significant differences between the groups were seen for changes in ePDT in the Ventral T10 dermatome $(H=5.14$, $P=0.162$ ) (Table 2 and Figure 4).

\section{Conditioned pain modulation}

CPM response was comparable among all the four patient groups at baseline. Within the groups, the pregabalin responders showed a significant increase in CPM response, ie, a more effective response, after 3 weeks of treatment $(P=0.006)$. In contrast, the placebo responders showed a nonsignificant trend for a decrease in CPM response, which is a less effective response (more pro-nociceptive pain modulation), after 3 weeks of treatment $(P=0.028)$ (Table 2).

The changes in CPM responses were significantly different among the groups $(H=11.3, P=0.01)$. Post hoc analysis showed that the pregabalin responders had a CPM response that differed significantly from that of the patients in the placebo responder group $(P<0.001)$ (Table 2 and Figure 5).

\section{Discussion}

This is the first study to describe the relation between the clinical analgesic response, defined as responders and nonresponders, to placebo or pregabalin treatment and changes in pain processing using QST measures in patients with chronic pancreatitis. Our study shows the presence of significant antihyperalgesic effects (decrease in pain sensitivity) in pregabalin responders and no significant anti-hyperalgesic effects in placebo responders. The pregabalin responders showed significantly more anti-hyperalgesic effects compared with pregabalin nonresponders. With treatment, pregabalin responders' descending pain modulation (CPM response) became significantly more inhibitory, whereas that of placebo responders showed a trend toward becoming more facilitatory.

Our results indicate that both underlying mechanisms and the placebo effect need to be taken into account in the clinical management of pain in chronic pancreatitis. Firstly, the response to placebo analgesia was not accompanied by significant anti-hyperalgesia. Thus, mechanisms other than antihyperalgesia appear to operate in placebo analgesia. Secondly, only in the pregabalin responders, pain thresholds within and distant from the pancreatic segment increased significantly. 
Table I Demographic and clinical characteristics of patients

\begin{tabular}{|c|c|c|c|c|}
\hline & $\begin{array}{l}\text { Placebo } \\
\text { responders }(n=12)\end{array}$ & $\begin{array}{l}\text { Placebo } \\
\text { nonresponders }(n=\mid 7)\end{array}$ & $\begin{array}{l}\text { Pregabalin } \\
\text { responders }(n=16)\end{array}$ & $\begin{array}{l}\text { Pregabalin } \\
\text { nonresponders }(n=\mid 5)\end{array}$ \\
\hline Age (years) & $54(4 I-63)$ & $59(49-64)$ & $52(50-59)$ & $49(43-57)$ \\
\hline Males, n (\%) & $9(75)$ & $10(59)$ & $9(56)$ & $9(60)$ \\
\hline \multicolumn{5}{|l|}{ Etiology, n (\%) } \\
\hline - Toxic, metabolic & $9(75)$ & $7(4 I)$ & $6(38)$ & $9(60)$ \\
\hline - Idiopathic & $2(17)$ & $9(53)$ & $6(38)$ & $5(33)$ \\
\hline - Genetic & $0(0)$ & $0(0)$ & $\mathrm{I}(6)$ & $\mathrm{I}(7)$ \\
\hline - Autoimmune & $0(0)$ & $0(0)$ & I (6) & $0(0)$ \\
\hline - Recurrent and severe acute pancreatitis & $0(0)$ & I (6) & I (6) & $0(0)$ \\
\hline - Obstructive & I (8) & $0(0)$ & $I(6)$ & $0(0)$ \\
\hline \multicolumn{5}{|l|}{ Diary pain score (VAS before) } \\
\hline - Average pain & $4(2-5)$ & $4(2-5)$ & $4(3-5)$ & $4(2-7)$ \\
\hline - Maximum pain & $5(2-7)$ & $5(4-7)$ & $6(4-9)$ & $7(4-8)$ \\
\hline \multicolumn{5}{|l|}{ Diary pain score (VAS after)* } \\
\hline - Average pain & I $(0-2)^{\#}$ & $4(2-6)$ & I $(0-2)^{\#}$ & $4(2-6)$ \\
\hline - Maximum pain & I (0-4)\# & $5(4-7)$ & $2(1-5)^{\#}$ & $5(4-7)$ \\
\hline \multicolumn{5}{|l|}{ Concomitant analgesics, $\mathrm{n}(\%)^{\dagger}$} \\
\hline - None & $2(17)$ & $0(0)$ & $2(13)$ & I (7) \\
\hline - Weak analgesics & $3(25)$ & $8(47)$ & $4(25)$ & $3(20)$ \\
\hline - Strong analgesics & $7(558)$ & $9(53)$ & $10(63)$ & II (73) \\
\hline $\mathrm{MEQ} / \mathrm{day}$ & $45(23-135)$ & $48(8-120)$ & $71(4-127)$ & $80(10-180)$ \\
\hline Duration of chronic pancreatitis (months) & $|5|(77-2 \mid 2)$ & $84(73-112)$ & $83(54-13 \mid)$ & $117(100-166)$ \\
\hline Diabetes mellitus, $\mathrm{n}(\%)$ & $6(50)$ & $4(24)$ & $7(44)$ & $3(20)$ \\
\hline \multicolumn{5}{|c|}{ Previous interventions for chronic pancreatitis, no (\%) } \\
\hline - Pancreas resection/drainage procedures & $2(17)$ & $3(18)$ & $3(19)$ & $3(20)$ \\
\hline - Thoracoscopic splanchnic denervation & I (8) & $3(18)$ & $0(0)$ & $2(13)$ \\
\hline - Celiac blockade & I (8) & $0(0)$ & $I(6)$ & $0(0)$ \\
\hline $\begin{array}{l}\text { Enzyme treatment for pancreatic exocrine } \\
\text { insufficiency, } \mathrm{n}(\%)\end{array}$ & $6(50)$ & $6(35)$ & $7(44)$ & $8(53)$ \\
\hline Ongoing alcohol abuse, $\mathrm{n}(\%)^{\ddagger}$ & $4(33)$ & $6(35)$ & $3(19)$ & $3(20)$ \\
\hline Current smoker, n (\%) & $9(75)$ & $13(76)$ & $12(80)$ & $12(75)$ \\
\hline
\end{tabular}

Notes: All values are medians with IQRs unless mentioned otherwise. Percentages may not total I00 due to rounding. VAS: 0-I0. tWeak analgesics were defined as NSAIDs, paracetamol, codeine, and tramadol. Strong analgesics were defined as opioid-based therapies. "Pregabalin" is pregabalin study group and "placebo" is placebo study group; *alcohol-abusing patients were defined as female patients drinking 14 units of alcohol per week or male patients drinking 2 I units of alcohol per week; \#a significant reduction in mean and maximum VAS scores was observed within the treatment group after study treatment $(P$-value $\leq 0.010)$; *statistical differences between groups where the placebo responders and pregabalin responders differed significantly $(P$-value $\leq 0.010)$ from the placebo nonresponders and pregabalin nonresponders.

Abbreviations: IQR, interquartile range; MEQ, morphine equivalents; NSAID, nonsteroidal anti-inflammatory drug; VAS, visual analog scale.

Table 2 Pain thresholds and CPM response at baseline, after 3 weeks' treatment and the change in values for all the groups

\begin{tabular}{|c|c|c|c|c|}
\hline & $\begin{array}{l}\text { Placebo } \\
\text { responders }(n=12)\end{array}$ & $\begin{array}{l}\text { Placebo } \\
\text { nonresponders }(n=\mid 7)\end{array}$ & $\begin{array}{l}\text { Pregabalin } \\
\text { responders }(n=16)\end{array}$ & $\begin{array}{l}\text { Pregabalin } \\
\text { nonresponders }(n=15)\end{array}$ \\
\hline \multicolumn{5}{|l|}{ ePDT (mA) (baseline) } \\
\hline$-\mathrm{C} 5$ & $3.5(2.4-7.6)$ & $3.5(2.2-5.5)$ & $3.8(2.7-4.7)$ & $3.6(2.2-5.1)$ \\
\hline$-\mathrm{TIOV}$ & $6.1(3.5-7.8)$ & $3.5(2.5-6.8)$ & $5.7(4.5-7.6)$ & $5.2(4.5-10.4)$ \\
\hline \multicolumn{5}{|l|}{ ePDT (after treatment) } \\
\hline$-\mathrm{C5}$ & $5.0(2.0-6.3)$ & $2.3(2.1-4.5)$ & $4.8(3.5-6.3)^{*}$ & $4.5(3.3-5.4)$ \\
\hline - TIOV & $7.0(5.0-8.2)$ & $3.4(3.0-6.1)$ & $6.8(5.8-10.1)^{\dagger}$ & $6.4(3.9-7.5)$ \\
\hline \multicolumn{5}{|l|}{ Change ePDT } \\
\hline$-\mathrm{C} 5$ & $-0.1(-1.3$ to 0.5$)$ & $-0.3(-0.7$ to 0.6$)$ & $1.3(0.4-2.0)$ & $0.4(-0.9$ to 1.3$)$ \\
\hline - TIOV & $0.7(-0.6$ to 1.9$)$ & $-0.2(0.8-1.2)$ & $1.7(0.0-3.4)$ & $-0.3(-1.1$ to 1.9$)$ \\
\hline CPM response (\%) (baseline) & $13(-3$ to 25$)$ & $14(5-44)$ & II $(-12$ to 23$)$ & $12(-19$ to 39$)$ \\
\hline CPM response (\%) (after treatment) & $-3(-12$ to 9$)$ & $17(-2$ to 36$)$ & $15(0-50)^{\ddagger}$ & $0(-1$ to 23$)$ \\
\hline Change in CPM response (\%) & $-17(-35$ to -6$)$ & $3(-22$ to 10$)$ & $9(4-55)$ & $-7(-35$ to 16$)$ \\
\hline
\end{tabular}

Notes: All values are medians with IQRs. C5 is the C5 dermatome (lower neck), and TIOV is the ventral TIO dermatome (upper abdominal area). Statistical difference between baseline and after treatment values: $* P=0.009,+P=0.009$, and $¥ P=0.006$.

Abbreviations: CPM, conditioned pain modulation; ePDT, electric pain detection thresholds; IQR, interquartile range. 


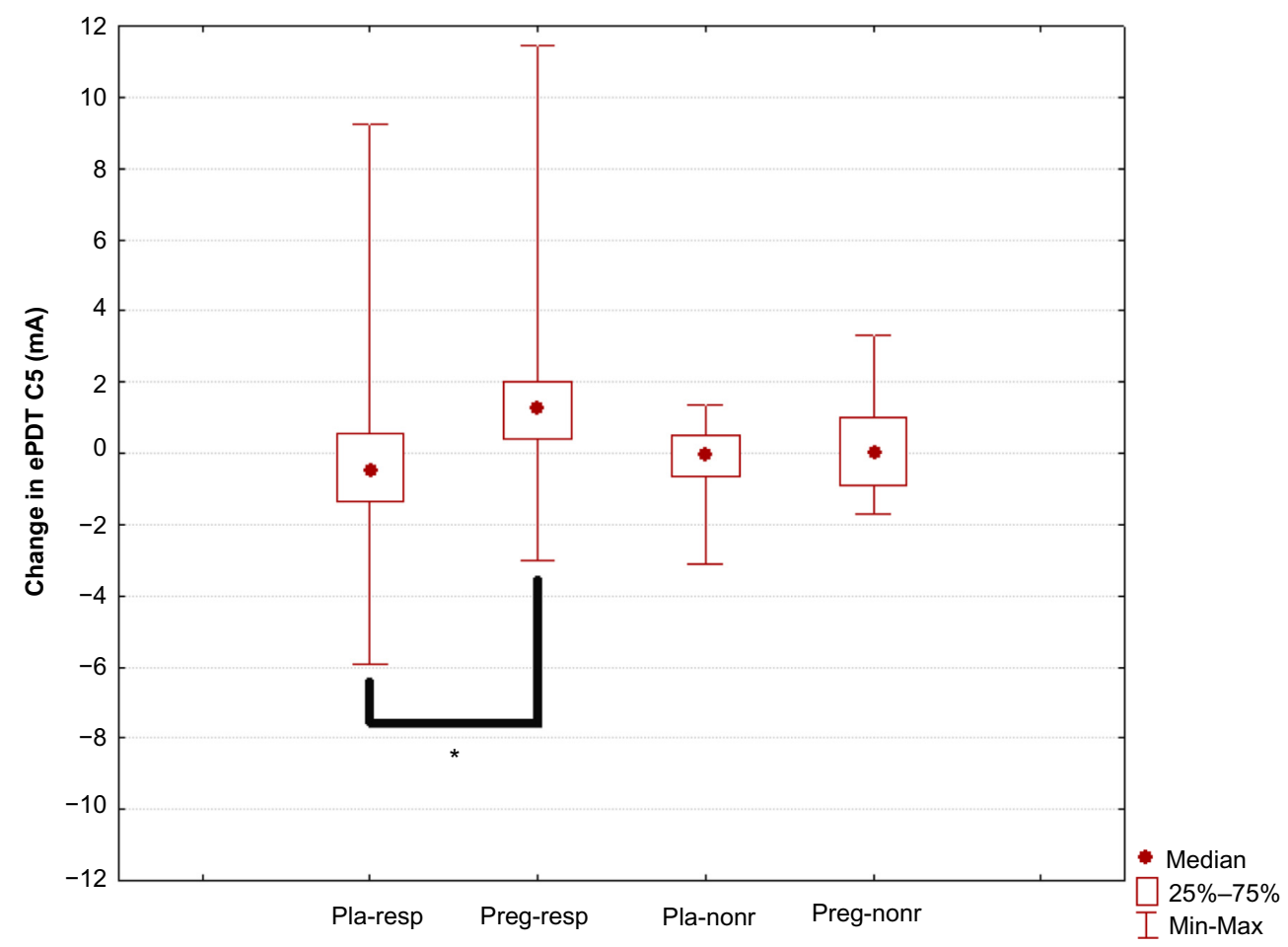

Figure 3 Change in electric pain detection thresholds at dermatome C5.

Notes: "Pla-resp" is the placebo responder group, "preg-resp" is the pregabalin responder group, "pla-nonr" is the placebo nonresponder group, and "preg-nonr" is the pregabalin nonresponder group. Significant differences are marked with an asterisk $(P=0.015)$.

Abbreviation: ePDT, electric pain detection thresholds.

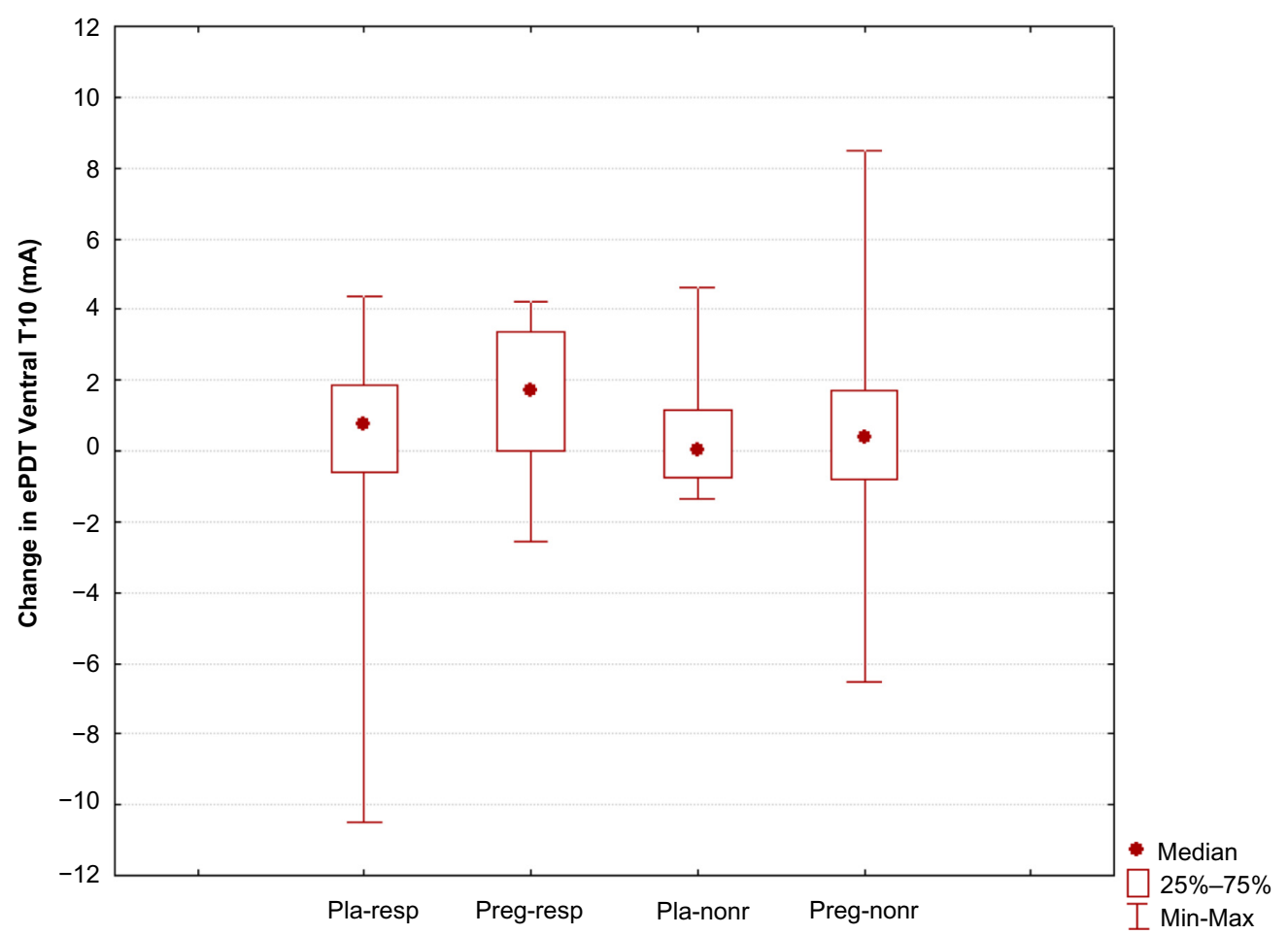

Figure 4 Change in electric pain detection thresholds at dermatome Ventral TIO.

Note: "Pla-resp" is the placebo responder group, "preg-resp" is the pregabalin responder group, "pla-nonr" is the placebo nonresponder group, and "preg-nonr" is the pregabalin nonresponder group.

Abbreviation: ePDT, electric pain detection thresholds. 


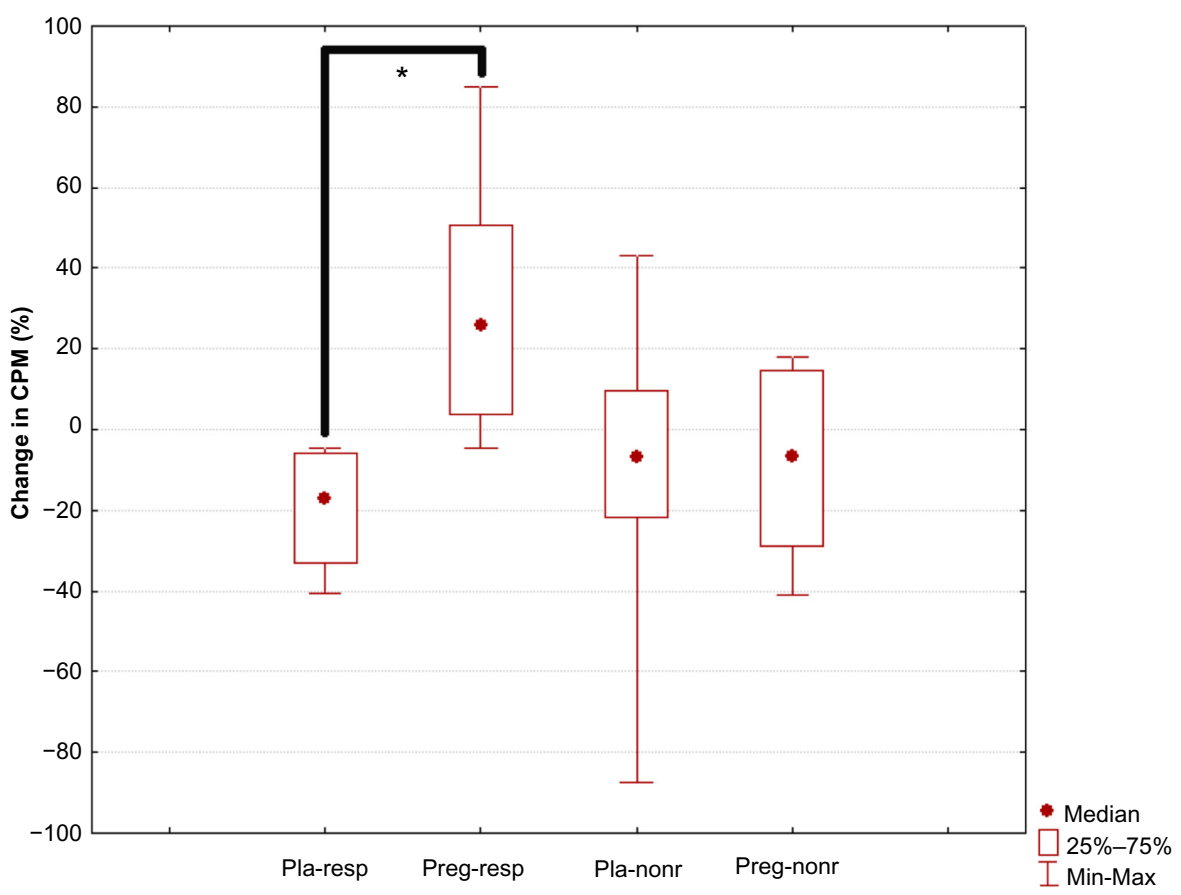

Figure 5 Change in conditioned pain modulation response.

Notes: "Pla-resp" is the placebo responder group, "preg-resp" is the pregabalin responder group, "pla-nonr" is the placebo nonresponder group, and "preg-nonr" is the pregabalin nonresponder group. Significant differences are marked with an asterisk $(P<0.00 \mathrm{I})$.

Abbreviation: CPM, conditioned pain modulation.

Distant from the pancreatic segment, this anti-hyperalgesic effect was significantly larger in the pregabalin responders than the nonresponders. In the pancreatic segment, the anti-hyperalgesic response to pregabalin treatment did not differ significantly between the pregabalin responders and nonresponders. This suggests that analgesic response to pregabalin treatment is linked to anti-hyperalgesic effect, although other mechanisms may also be operating. Furthermore, generalized anti-hyperalgesic effects appear more prominent than segmental anti-hyperalgesic effects. Thirdly, it would appear that endogenous descending pain modulation plays an important role in analgesic response and may be fundamentally different for placebo vs pregabalin treatments.

\section{Effect of pregabalin}

Treatments targeting the central nervous system, eg, gabapentinoids or tricyclic antidepressants, are increasingly being demonstrated to improve the management of chronic pain disorders. ${ }^{26}$ For chronic pancreatitis, we have shown pregabalin to be effective in reducing pain scores after 3 weeks of treatment together with a positive overall treatment response as rated by patients. ${ }^{4}$ Accompanying the clinical effect, we found an anti-hyperalgesic effect of pregabalin for electric stimuli. ${ }^{6}$ The present study further elucidates these results by showing that anti-hyperalgesic effects in responders to pregabalin are significant, particularly in a dermatome distant from the pancreas. The overall size of the effect in the pancreatic segment was comparable between the pregabalin responders and nonresponders. In another study, we showed that patients with chronic pancreatitis with segmental hyperalgesia had a superior clinical response to pregabalin. ${ }^{27}$ Taken together, these results suggest that the major anti-hyperalgesic effect of pregabalin is on ascending spinal sensitization rather than directly on sensitization within the pancreatic spinal segment. Clearly, further research is needed regarding the sites of action of pregabalin within the nervous system. Although baseline CPM values were similar, modulatory responses were greater and significantly inhibitory in pregabalin (vs placebo) responders. This outcome could be construed as a direct, anti-facilitatory effect of pregabalin on CPM, or an indirect one based on the reduction of ascending central nociceptive transmission. ${ }^{22}$ If pregabalin affects ascending central pathways, it might also affect descending ones, an explanation that could support direct effects on CPM. A further possible explanation could be other differences between the pregabalin responder and nonresponder patients, ie, regarding the use of more strong analgesics or a history of more pain reducing previous interventions.

Studies on the effects of centrally active medication, eg, pregabalin, gabapentin, or S-ketamine, on central sensitization are limited..$^{18,28-33}$ They demonstrate reduction 
of hyperalgesia (central sensitization), in accordance with our study. None of these studies investigated differences between placebo and drug responders, and none studied pain modulation (CPM). Responders and nonresponders to gabapentin have been described in a rat study, where rats with selective nerve injury (spinal level) showed differences in descending inhibition. The extent of suggested changes in the central nervous system may predict the effect of pregabalin and gabapentin treatment. ${ }^{34}$

\section{Effect of placebo}

The placebo effect on clinical pain has been described extensively. ${ }^{35-37}$ Imaging studies of the brain have shown increased activity in pain-related areas during painful stimuli and placebo analgesia. ${ }^{38} \mathrm{~A}$ relation has been demonstrated between desire for pain relief or expected pain levels and opioid-related activity in certain brain areas. These psychological factors and placebo effect can be antagonized by naloxone. ${ }^{39}$ The effect of placebo analgesia appears to be highly variable and depends on contextual factors. ${ }^{11,40,41}$ In our clinical study, we found a strong placebo response, for the whole group, albeit significantly lower than that with pregabalin treatment. ${ }^{4}$ This placebo response may be explained by the psychosocial context and in the suggestion of treatment effect. $^{36,37,42}$ Patients with chronic pancreatitis, with their extensive medical history, their expectation or desire for pain relief, and the possibility of being randomized for pregabalin, may be particularly susceptible to placebo effects.

Our study showed that the placebo responders had a significant strong clinical effect on clinical pain compared with the placebo nonresponders. However, we also demonstrated no significant anti-hyperalgesic effects for both the responders and nonresponders to placebo. As effects on skin pain sensitivity were similar in the placebo responders and nonresponders, central anti-hyperalgesia is unlikely to be the mechanism underlying placebo analgesia, at least in patients with chronic pancreatitis. Furthermore, a trend for a decrease in inhibitory CPM response was seen only in the placebo responders. Theoretically, this finding could be linked to a negative interaction of placebo effect with CPM. This unexpected trend result further suggests that anti-hyperalgesia is not a major mechanism underlying analgesia in this context. Clearly, more research is necessary.

\section{Limitations}

A limitation of this study is the relatively small size of the four patient groups. Nevertheless, significant differences could be observed among the groups. Furthermore, we may have introduced bias by using a per-protocol analysis. However, we think that analyzing only the patients who fully complied to the study protocol, ie, used all their study medications and had measurements after study treatment, is the proper way to investigate mechanisms in responders and nonresponders to the trial medication. It should be noted that the majority of the patients in both the study arms were compliant and all study medications were taken correctly. The duration of study medication in the initial trial was 3 weeks; perhaps, a longer study period would have given a more distinct difference between both the groups. In our clinical study, 39\% of pregabalin patients did not tolerate $300 \mathrm{mg}$ pregabalin twice daily, and were treated with $150 \mathrm{mg}$ twice daily. The distribution of patients not tolerating $300 \mathrm{mg}$ twice daily was not significantly different between the pregabalin responders and nonresponders. Perhaps, in a larger population, this difference could be of significance. Another source of bias could have been that the groups were not comparable at baseline for factors we did not measure, ie, expectations regarding study effect or other psychological measures. However, this seems unlikely because no significant differences could be found in the clinically relevant baseline characteristics among the four groups. Moreover, no differences in baseline values were found for use of strong analgesics, morphine equivalents, prevalence of diabetes mellitus, nicotine exposure, or alcohol consumption (possible confounding effects on pain perception) among all the four groups.

Because chronic pancreatitis is predominantly a visceral pain syndrome, we could have introduced bias by only analyzing electric stimulation (somatic skin pain) and not pressure stimulation (deep tissue and visceral pain) in this study. The reason we chose to analyze only electrical stimulation was based on earlier study results, ${ }^{6}$ where electrical stimulation was the earliest and most sensitive parameter of change in pain sensitization due to medication in patients with chronic pancreatitis. In this study, we found that the anti-hyperalgesic effect of pregabalin resulted in a greater increase of electric pain thresholds than that of pressure pain thresholds after 3 weeks of treatment. ${ }^{6}$ This is probably because the skin, in comparison with deep and visceral tissue, is a tissue that is constantly and highly subject to sensory modulation by the central nervous system. Thus, changes in central sensitization will likely first be reflected in skin projection areas of the spinal dorsal horn neurons sensitized by the chronic pancreatitis. ${ }^{43}$

\section{Conclusion}

Our study provides evidence that patients treated with placebo show no anti-hyperalgesic effects paralleling pain relief 
after a treatment period of 3 weeks in chronic pancreatitis. Patients with a positive effect on clinical pain following pregabalin treatment were the only ones to show significant anti-hyperalgesic effects, particularly in the dermatome distant from the pancreas, with comparable anti-hyperalgesic effects for pregabalin responders and nonresponders in the pancreatic dermatome itself. The pregabalin responders showed an increase in endogenous inhibitory modulation compared with a trend to decrease in the placebo responders. Both the existence of a potent placebo effect and the difference between responders and nonresponders regarding underlying pain sensitivity and modulation need to be taken into account in the management of pain in chronic pancreatitis.

\section{Acknowledgment}

Our study was supported by a free grant from Pfizer Research and Development, Hertha Christensen's Foundation, and Christenson-Ceson's Family Foundation. The study was investigator initiated and Pfizer donated capsules of pregabalin and placebo. The funders had no role in study design, data collection and analysis, decision to publish, or preparation of the manuscript.

\section{Disclosure}

The authors report no conflicts of interest in this work.

\section{References}

1. Steer ML, Waxman I, Freedman S. Chronic pancreatitis. N Engl J Med. 1995;332(22):1482-1490.

2. Andren-Sandberg A, Hoem D, Gislason H. Pain management in chronic pancreatitis. Eur J Gastroenterol Hepatol. 2002;14(9):957-970.

3. van Esch AA, Wilder-Smith OH, Jansen JB, van Goor H, Drenth JP. Pharmacological management of pain in chronic pancreatitis. Dig Liver Dis. 2006;38(7):518-526.

4. Olesen SS, Bouwense SA, Wilder-Smith OH, van Goor H, Drewes AM. Pregabalin reduces pain in patients with chronic pancreatitis in a randomized, controlled trial. Gastroenterology. 2011;141(2):536-543.

5. Bouwense SA, Olesen SS, Drewes AM, Frokjaer JB, van Goor H, Wilder-Smith $\mathrm{OH}$. Is altered central pain processing related to disease stage in chronic pancreatitis patients with pain? An exploratory study. PloS One. 2013;8(2):e55460.

6. Bouwense SA, Olesen SS, Drewes AM, Poley JW, van Goor H, WilderSmith $\mathrm{OH}$. Effects of pregabalin on central sensitization in patients with chronic pancreatitis in a randomized, controlled trial. PloS One. 2012;7(8):e42096.

7. Capurso G, Cocomello L, Benedetto U, Camma C, Delle Fave G. Meta-analysis: the placebo rate of abdominal pain remission in clinical trials of chronic pancreatitis. Pancreas. 2012;41(7): $1125-1131$

8. Price DD, Craggs J, Verne GN, Perlstein WM, Robinson ME. Placebo analgesia is accompanied by large reductions in pain-related brain activity in irritable bowel syndrome patients. Pain. 2007;127(1-2): 63-72.

9. Vase L, Robinson ME, Verne GN, Price DD. The contributions of suggestion, desire, and expectation to placebo effects in irritable bowel syndrome patients. An empirical investigation. Pain. 2003;105(1-2): $17-25$.
10. Vase L, Robinson ME, Verne GN, Price DD. Increased placebo analgesia over time in irritable bowel syndrome (IBS) patients is associated with desire and expectation but not endogenous opioid mechanisms. Pain. 2005;115(3):338-347.

11. Amanzio M, Pollo A, Maggi G, Benedetti F. Response variability to analgesics: a role for non-specific activation of endogenous opioids. Pain. 2001;90(3):205-215.

12. Levine JD, Gordon NC, Fields HL. The mechanism of placebo analgesia. Lancet. 1978;2(8091):654-657.

13. Zubieta JK, Bueller JA, Jackson LR, et al. Placebo effects mediated by endogenous opioid activity on mu-opioid receptors. $J$ Neurosci. 2005;25(34):7754-7762.

14. Olesen SS, Graversen C, Bouwense SA, van Goor H, Wilder-Smith OH, Drewes AM. Quantitative sensory testing predicts pregabalin efficacy in painful chronic pancreatitis. PloS One. 2013;8(3):e57963.

15. Cui JG, O'Connor WT, Ungerstedt U, Linderoth B, Meyerson BA. Spinal cord stimulation attenuates augmented dorsal horn release of excitatory amino acids in mononeuropathy via a GABAergic mechanism. Pain. 1997;73(1):87-95.

16. Song Z, Ultenius C, Meyerson BA, Linderoth B. Pain relief by spinal cord stimulation involves serotonergic mechanisms: an experimental study in a rat model of mononeuropathy. Pain. 2009;147(1-3): 241-248.

17. Woolf CJ, Thompson SW. The induction and maintenance of central sensitization is dependent on N-methyl-D-aspartic acid receptor activation; implications for the treatment of post-injury pain hypersensitivity states. Pain. 1991;44(3):293-299.

18. Bouwense SA, Buscher HC, van Goor H, Wilder-Smith OH. S-ketamine modulates hyperalgesia in patients with chronic pancreatitis pain. Reg Anesth Pain Med. 2011;36(3):303-307.

19. Bouwense SA, Buscher HC, van Goor H, Wilder-Smith OH. Has central sensitization become independent of nociceptive input in chronic pancreatitis patients who fail thoracoscopic splanchnicectomy? Reg Anesth Pain Med. 2011;36(6):531-536.

20. Layer P, Yamamoto H, Kalthoff L, Clain JE, Bakken LJ, DiMagno EP. The different courses of early- and late-onset idiopathic and alcoholic chronic pancreatitis. Gastroenterology. 1994;107(5):1481-1487.

21. Buscher HC, Wilder-Smith OH, van Goor H. Chronic pancreatitis patients show hyperalgesia of central origin: a pilot study. Eur J Pain. 2006;10(4):363-370.

22. Pud D, Granovsky Y, Yarnitsky D. The methodology of experimentally induced diffuse noxious inhibitory control (DNIC)-like effect in humans. Pain. 2009;144(1-2):16-19.

23. Nir RR, Granovsky Y, Yarnitsky D, Sprecher E, Granot M. A psychophysical study of endogenous analgesia: the role of the conditioning pain in the induction and magnitude of conditioned pain modulation. Eur J Pain. 2011;15(5):491-497.

24. Farrar JT, Young JP Jr, LaMoreaux L, Werth JL, Poole RM. Clinical importance of changes in chronic pain intensity measured on an 11-point numerical pain rating scale. Pain. 2001;94(2):149-158.

25. Dworkin RH, Turk DC, Wyrwich KW, et al. Interpreting the clinical importance of treatment outcomes in chronic pain clinical trials: IMMPACT recommendations. J Pain. 2008;9(2):105-121.

26. Finnerup NB, Sindrup SH, Jensen TS. The evidence for pharmacological treatment of neuropathic pain. Pain. 2010;150(3): 573-581.

27. Olesen SS, van Goor H, Bouwense SA, Wilder-Smith OH, Drewes AM. Reliability of static and dynamic quantitative sensory testing in patients with painful chronic pancreatitis. Reg Anesth Pain Med. 2012;37(5): 530-536.

28. Bornemann-Cimenti H, Lederer AJ, Wejbora M, et al. Preoperative pregabalin administration significantly reduces postoperative opioid consumption and mechanical hyperalgesia after transperitoneal nephrectomy. Br J Anaesth. 2012;108(5):845-849.

29. Zhang N, Chen CF, Yu FY. Effects of pregabalin on central sensitization in patients with migraine. Int J Clin Pharmacol Ther. 2015;53(4): 277-283. 
30. Zhang Y, Shao G, Zhang W, et al. Gabapentin inhibits central sensitization during migraine. Neural Regen Res. 2013;8(32):3003-3012.

31. Houghton LA, Fell C, Whorwell PJ, Jones I, Sudworth DP, Gale JD. Effect of a second-generation alpha2delta ligand (pregabalin) on visceral sensation in hypersensitive patients with irritable bowel syndrome. Gut. 2007;56(9):1218-1225.

32. Arendt-Nielsen L, Frokjaer JB, Staahl C, et al. Effects of gabapentin on experimental somatic pain and temporal summation. Reg Anesth Pain Med. 2007;32(5):382-388.

33. Gottrup H, Juhl G, Kristensen AD, et al. Chronic oral gabapentin reduces elements of central sensitization in human experimental hyperalgesia. Anesthesiology. 2004;101(6):1400-1408.

34. Suzuki R, Rahman W, Rygh LJ, Webber M, Hunt SP, Dickenson AH. Spinal-supraspinal serotonergic circuits regulating neuropathic pain and its treatment with gabapentin. Pain. 2005;117(3):292-303.

35. Hrobjartsson A, Gotzsche PC. Is the placebo powerless? An analysis of clinical trials comparing placebo with no treatment. New Engl J Med. 2001;344(21):1594-1602.

36. Vase L, Petersen GL, Riley JL III, Price DD. Factors contributing to large analgesic effects in placebo mechanism studies conducted between 2002 and 2007. Pain. 2009;145(1-2):36-44.
37. Vase L, Riley JL 3rd, Price DD. A comparison of placebo effects in clinical analgesic trials versus studies of placebo analgesia. Pain. 2002; 99(3):443-452.

38. Elsenbruch S, Kotsis V, Benson S, et al. Neural mechanisms mediating the effects of expectation in visceral placebo analgesia: an fMRI study in healthy placebo responders and nonresponders. Pain. 2012;153(2): 382-390.

39. Sauro MD, Greenberg RP. Endogenous opiates and the placebo effect: a meta-analytic review. J Psychosom Res. 2005;58(2):115-120.

40. Amanzio M, Benedetti F. Neuropharmacological dissection of placebo analgesia: expectation-activated opioid systems versus conditioningactivated specific subsystems. J Neurosci. 1999;19(1):484-494.

41. Colloca L, Benedetti F. How prior experience shapes placebo analgesia. Pain. 2006;124(1-2):126-133.

42. Hrobjartsson A, Gotzsche PC. Unsubstantiated claims of large effects of placebo on pain: serious errors in meta-analysis of placebo analgesia mechanism studies. J Clin Epidemiol. 2006;59(4):336-338.

43. Drewes AM, Krarup AL, Detlefsen S, Malmstrom ML, Dimcevski G, Funch-Jensen P. Pain in chronic pancreatitis: the role of neuropathic pain mechanisms. Gut. 2008;57(11):1616-1627. 


\section{Supplementary material}

The present study is a hypothesis-generating and an exploratory analysis of experimental end points of our clinical trial using pregabalin and placebo in chronic pancreatitis. ${ }^{1}$ One of our earlier studies describing these experimental end points showed that our patients with chronic pancreatitis were hyperalgesic, and this could be related to disease stage based on the Mannheim severity index. ${ }^{2}$ Later, we described the effect of pregabalin on pain processing in chronic pancreatitis. Only the patients randomized for pregabalin were analyzed, and it was shown that pregabalin has moderate inhibitory effects on central sensitization, manifest as spreading hyperalgesia in patients with chronic pancreatitis. ${ }^{3}$ In another study, we examined whether quantitative sensory testing (QST) could predict the clinical effect of pregabalin and placebo. Study results showed that QST predicts the analgesic effect of pregabalin in patients with painful chronic pancreatitis. ${ }^{4}$ Another substudy of our clinical trial described end points based on or analyzed with electroencephalography and QST.5,6

What makes the present study different from the previously performed studies?

1. We described responders and nonresponders to placebo or pregabalin treatment.
2. We did not aim at clinical end points, ie, pain relief, but at an experimental end point, pain processing.

3. We investigated the difference in pain sensitivity and modulation by QST before and after study treatment, together with the change in pain sensitivity and modulation.

\section{References}

1. Olesen SS, Bouwense SA, Wilder-Smith OH, van Goor H, Drewes AM. Pregabalin reduces pain in patients with chronic pancreatitis in a randomized, controlled trial. Gastroenterology. 2011;141(2):536-543.

2. Bouwense SA, Olesen SS, Drewes AM, Frokjaer JB, van Goor H, Wilder-Smith $\mathrm{OH}$. Is altered central pain processing related to disease stage in chronic pancreatitis patients with pain? An exploratory study. PloS One. 2013;8(2):e55460.

3. Bouwense SA, Olesen SS, Drewes AM, Poley JW, van Goor H, WilderSmith $\mathrm{OH}$. Effects of pregabalin on central sensitization in patients with chronic pancreatitis in a randomized, controlled trial. PloS One. 2012;7(8):e42096.

4. Olesen SS, Graversen C, Bouwense SA, van Goor H, Wilder-Smith OH, Drewes AM. Quantitative sensory testing predicts pregabalin efficacy in painful chronic pancreatitis. PloS One. 2013;8(3):e57963.

5. Graversen C, Olesen SS, Olesen AE, et al. The analgesic effect of pregabalin in patients with chronic pain is reflected by changes in pharmacoEEG spectral indices. Br J Clin Pharmacol. 2012;73(3):363-372.

6. Olesen SS, Graversen C, Olesen AE, et al. Randomised clinical trial: pregabalin attenuates experimental visceral pain through sub-cortical mechanisms in patients with painful chronic pancreatitis. Aliment Pharmacol Ther. 2011;34(8):878-887.
Journal of Pain Research

\section{Publish your work in this journal}

The Journal of Pain Research is an international, peer-reviewed, open access, online journal that welcomes laboratory and clinical findings in the fields of pain research and the prevention and management of pain. Original research, reviews, symposium reports, hypothesis formation and commentaries are all considered for publication.

\section{Dovepress}

The manuscript management system is completely online and includes a very quick and fair peer-review system, which is all easy to use. Visit http://www.dovepress.com/testimonials.php to read real quotes from published authors. 